\title{
Dynamic acyclic motion from a planar contact-stance to another
}

\author{
Mario Arbulu, Kazuhito Yokoi, Abderrahmane Kheddar and Carlos Balaguer
}

\begin{abstract}
This paper addresses the problem of generating dynamic motion for a humanoid between two predefined postures. The humanoid robot starts its motion from a statically stable configuration to another known statically stable configuration when it is necessary to make step; that is, the center of mass (COM) is inside the support polygon at the initial and the goal configurations. A dynamic motion generation method is proposed in order to generate the whole-body robot motion. Zero moment point preview control is used for maintaining dynamic stability while the robot is taking the step and suitable splines are used for generating the hands, neck and foot motion because it is a free motion on the field of gravity; the screw theory is applied under Lie groups for kinematics and dynamics humanoid modeling, which allows smooth and natural humanoid motion. Successful results on the HRP-2 humanoid robot are shown and discussed.
\end{abstract}

\section{INTRODUCTION}

The gait motion planning for humanoid robot in order to walk on a flat surface has been solved with various approaches. Many take into account the concentrated mass model [1] and others the distributed mass model, furthermore considering friction [2]. The first work ([1]) does not deal with the effect of whole-body motion during humanoid walking motion. In a cyclic motion patterns could be generated at real-time, but the dynamic effect of the whole-body inertial forces and gravity are compensated by the controller. The second work ([2]) deals with whole-body motion while walking and accounts for a strong stability criteria (the sum of the contact wrench should be inside the contact wrench cone); it is a more accurate model but the high computation cost and the iterative solution does not make it possible to generate the patterns in real time; successful simulation have been obtained. To go from any posture to another by a step (which we call "acyclic gait"), the whole-body motion should be taken into account in order to maintain the dynamic stability but under constraints of realizing the desired posture configuration at the initial and final key-postures. Many works about humanoid robot whole-body motion have been carried out, some dealing with the control of linear and angular momentum [3],[4],[5]. The approach used in spatial

This work was supported by the University Carlos III of Madrid. M. Arbulu is with Department of Systems and Automation Engineering, Roboticslab, University Carlos III of Madrid, Av. de la Universidad 30 , 28911, Leganes, Madrid, Spain marbulu@ing.uc3m.es

C. Balaguer is with Department of Systems and Automation Engineering, Roboticslab, University Carlos III of Madrid, Av. de la Universidad 30 28911, Leganes, Madrid, Spain balaguer@ing.uc3m.es

A. Kheddar is with AIST-CNRS Joint Japanese-French Robotics Laboratory JRL AIST Tsukuba Central 2, Umezono 1-1-1, Tsukuba 305-8568 Japan kheddar@ieee.org

$\mathrm{K}$. Yokoi is with AIST-CNRS Joint Japanese-French Robotics Laboratory JRL AIST Tsukuba Central 2, Umezono 1-1-1, Tsukuba 305-8568 Japan Kazuhito.Yokoi@aist.go.jp robotics [6], provides an interesting whole-body motion control by controlling the Linear and Angular momentum. From the point-of-view of high redundancy dividing the wholebody motion in tasks taking into account external forces acting on the robot, Khatib et. al. [7], Sentis et. al. [8], Mansard and Chaumette [9]. Path planning methods are an alternative to generate whole body motion [23], especially when there are obstacles to be avoided. These works divide whole-body motion into tasks and create a hierarchy. Other criterions at the kinematic level, are proposed by Yoshida et. al. [10], when the hierarchy of tasks is obtained by the combination of joint selection matrix and the projection in the null-space. The dynamic stability while walking is obtained by ZMP preview control [11], [12], [13], [14]; whole-body motion is developed by the generalized inverse kinematics under the Jacobian method. We propose an approach by solving the whole-body motion in a direct and natural way by making use of Lie groups under the screw theory [15], [16].

The main contributions of this paper are: Propose and validate the acyclic gait algorithm for humanoid robots. Implement and validate the use of screws, which provide a geometric description of rigid motion, so the analysis of mechanism is greatly simplified. Avoid singularities because the global description of the rigid body motion; only is necessary to define two frames (base and tool) and the rotation axis of each DoF, for analyzing the kinematics in a closed way. The Paden-Kahan sub problems allow computing the inverse kinematics at position level. Faster computation time of the inverse kinematics respect to inverse jacobian method, Euler angles or D-H parameters, so it contributes to real time applications. For computing the Jacobian (dynamic analysis) is not necessary to derivate. Validate the ZMP preview control [11], for generating the acyclic gait motion. Propose and validate the "Local Axis Gait" algorithm [19], for generation of the local motion planning. This work is divided as follows: in the section II we state the problem; in section III the proposed solution is detailed; in section IV the results are shown and discussed, as an application in the HRP-2 humanoid robot [17], and finally the conclusions and the future works will be explained in section V.

\section{PROBLEM STATEMENT}

We aim at achieving whole body motion of a humanoid robot between any two statically stable contacts and posture (joints) configurations. In this stage, footprints occur on the plan. For each contact, key-posture static stability is obtained simply by projecting the center of mass (COM) position into the support polygon. Therefore, initial and final whole-body configuration, that is: the feet, hip, hands and neck positions 


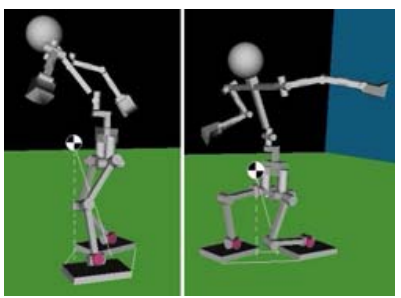

Fig. 1. Motion from any statically stable posture to other one.

and orientation are also known (Fig. 1). Stable dynamic motion is obtained by imposing to the ZMP [18] to be inside the support polygon. Note that, the boundary conditions (keypostures) are known but the transition behavior from the initial contact state to the other, is not defined. This work proposes an approach that realizes the dynamic transition behavior.

\section{OVERVIEW OF THE METHOD}

Our method make use of a combination of the following main components (Fig. 2): (i) the ZMP-previous control [19] that will be adjusted to our problem for dynamic stable transition, (ii) the kinematics and dynamics modelling under Lie groups, (iii) Paden-Kahan sub-problems and screw theory [15], [16], [21]. Those theories allow us to compute the inverse kinematics directly without internal singularities and dynamics modelling, [22] by Jacobian manipulator.

The input values to our problem are the initial and goal robot configuration (joint states since two successive contact configuration have at least a non empty sub-set of common contacts). At first, we use the forward kinematics in order to compute the footprints and COM initial and goal locations. The "Local axis gait algorithm [19]" is used for the motion planning which takes into account constraints such as joint angular limits, and torque limits. The dynamic stable motion is obtained by the ZMP-Preview control [11]. Using inverse kinematics, the joint legs' motions are computed. Next, the inverse dynamics by the Lagrangian formulation is computed to assess for the joint torques of the dynamic motion.

1) Solving the forward kinematics problem: Forward kinematics is used in order to compute the COM and feet locations at initial and goal key-postures. By exploiting natural humanoid left-right symmetry, (Fig. 3.a). The first six DoF correspond to the position and orientation of the foot; the next six DoF correspond to the leg joints. Let $\mathbf{S}$ be a frame attached to the base system (support foot) and $\mathbf{T}$ be a frame attached to the humanoid COM. The reference configuration of the manipulator is $\mathbf{g s t}(\mathbf{0})$. Thus, the product of exponentials formula for the right leg forward kinematics is $\mathrm{g}_{s t}(\theta)$, with $\xi^{\wedge}$ the $4 \times 4$ matrices called "twists".

$$
g_{s t}(\theta)=e^{\xi_{1}^{\wedge} \theta_{1}} e^{\xi_{2}^{\wedge} \theta_{2}} e^{\xi_{3}^{\wedge} \theta_{3}} \cdots e^{\xi_{12}^{\wedge} \theta_{12}} g_{s t}(0)
$$

2) Lower body motion planning using " $L A G$ ” Algorithm: The COM motion planning is obtained by the ZMP Preview Control [11], and the foot motion is planned by using a

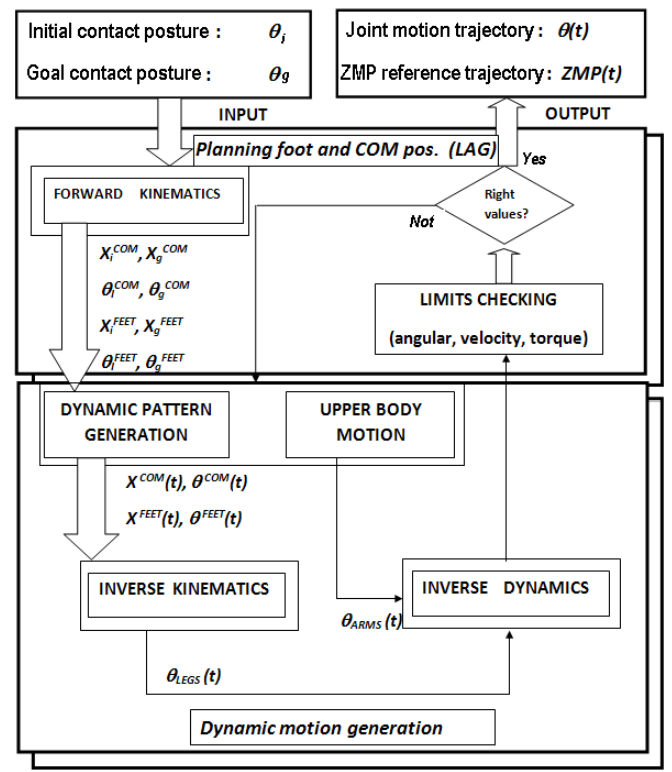

Fig. 2. Flowchart of the method. $X_{i}, X_{g}$, are the initial and goal position, and $\theta_{i}, \theta_{g}$ are the initial and goal orientation. Those are computed for the COM and feet by the forward kinematics. $X^{C O M}(t), X^{F E E T}(t)$ are the COM and FEET spatial trajectories, and $\theta^{C O M}(t), \theta^{F E E T}(t)$ are the $\mathrm{COM}$ and FEET orientation trajectories. $\theta_{L E G S}(t), \theta_{A R M S}(t)$ are the legs and arms joint trajectories.

$5^{\text {th }}$ order Splines. The Local Axis Gait (LAG) algorithm (Fig. 3.b) deals with local humanoid motion, which is to generate the $n^{\text {th }}$ step in any direction over any surface. The footprints (eq. 2) for doing a $n^{\text {th }}$ step, can be computed as follows:

$$
P^{n}=P^{n-1}+R\left(\theta_{z}^{n}\right)^{T} \cdot D^{n}
$$

where,

$$
P^{n}=\left(\begin{array}{ccc}
p_{x}^{n} & p_{y}^{n} & p_{z}^{n}
\end{array}\right)^{T}, D^{n}=\left(\begin{array}{ccc}
D_{x}^{n} & -(-1)^{n} D_{y}^{n} & D_{z}^{n}
\end{array}\right)^{T},
$$$$
P^{n}, P^{n-1}, P^{n+1} \text { are feet positions; } D_{x}^{n+1}, D_{y}^{n+1}, D_{z}^{n+1}
$$
are swing foot displacements; $\theta_{x}^{n+1}, \theta_{y}^{n+1}, \theta_{z}^{n+1}$ are rotations about world frame.

3) COM generation by ZMP Preview Control: There is one difference between the walking motion when the COM is moving in a plane. In Fig. 4, the preview gain for two differents vetical positions of the COM are shown. Thus, it is possible to consider the preview gain for the medium vertical position value (the value for $\overline{Z_{c}}$ ). For several simulation and experiments, it appears that is possible to consider the preview gain for the medium vertical position value (i. e. the value for $\overline{Z_{c}}$ ) variation of the parameters are tackled by the closed loop nature of the ZMP preview controller.

4) Solving the inverse kinematics problem: The inverse kinematics problem for the right leg (see Fig. 3) consists of finding the joint angles, $\left(\theta_{7}\right.$ to $\left.\theta_{12}\right)$. Using the product of exponentials (PoE) formula for the forward kinematics (eq. 


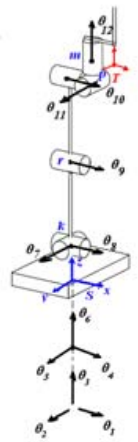

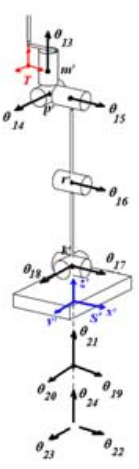

(a)

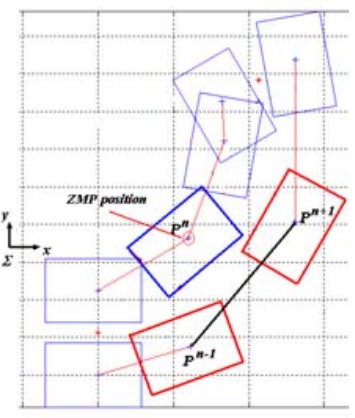

(b)
Fig. 3. (a) Sagittal Kinematics Division and (b) Footprints location.

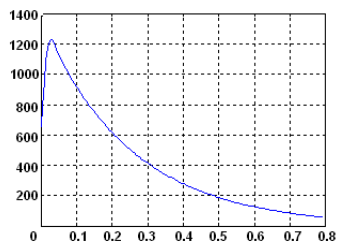

(a)

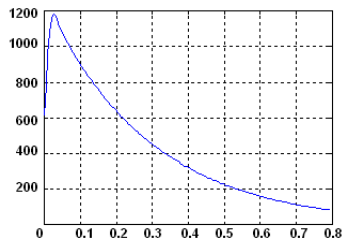

(b)
Fig. 4. Preview gain (at preview time of 0.8 seconds) $G_{p}(j)$ : (a) $Z_{c}=0.614 \mathrm{~m}$, (b) $Z_{c}=0.814 \mathrm{~m}$, when the $\mathrm{COM}$ is in lower position, the maximun value of the preview gain is higher with respect to the other one; so, the $u(k)$ value increase for walking at lower hip height.

(1)) it is possible to develop a numerically stable geometric algorithm to solve this problem, by using the Paden-Kahan (P-K) geometric subproblems:

$$
\begin{gathered}
\delta=\left\|e^{\xi_{9}^{\wedge} \theta_{9}} g_{s t}(0) \cdot p-k\right\| \stackrel{P-K-3}{\rightarrow} \theta_{9} \rightarrow \text { double sol. } \\
q^{\prime}=e^{\xi_{7}^{\wedge} \theta_{7}} \cdot e^{\xi_{8}^{\wedge} \theta_{8}} \cdot p^{\prime} \stackrel{P-K-2}{\rightarrow} \theta_{7}, \theta_{8} \rightarrow \text { double sol. } \\
q^{\prime \prime}=e^{\xi_{10}^{\wedge} \theta_{10}} \cdot e^{\xi_{11}^{\wedge} \theta_{11}} \cdot m \stackrel{P-K-2}{\longrightarrow} \theta_{10}, \theta_{11} \rightarrow \text { double sol. } \\
q^{\prime \prime \prime}=e^{\xi_{12}^{\wedge} \theta_{12}} \cdot S \stackrel{P-K-1}{\longrightarrow} \theta_{12} \rightarrow \text { single sol. }
\end{gathered}
$$

The upper body joint motion is obtained by suitable $7^{\text {th }}$ order Splines.

5) Solving the inverse dynamic problem: In order to compute the joint torques and dynamics constraints we use the Lagrange formulation under the lie groups and screw theory. This is because it gives a geometric description of a Jacobian manipulator [20], so for the right leg:

$$
J_{t l i}^{T}(\theta)=\left[\xi_{12}^{\dagger} \cdots \xi_{i}^{\dagger} \cdots 0 \cdots 0\right]
$$

\section{RESULTS}

Many tests have been done on the HRP-2 humanoid robot [17] in order to assess the proposed method. Some of these experiments are presented as follows: one acyclic step forward, one acyclic step backward with swing foot rotation and two acyclic steps forward. Note that the final and initial posture of the upper body is also different and can be any.

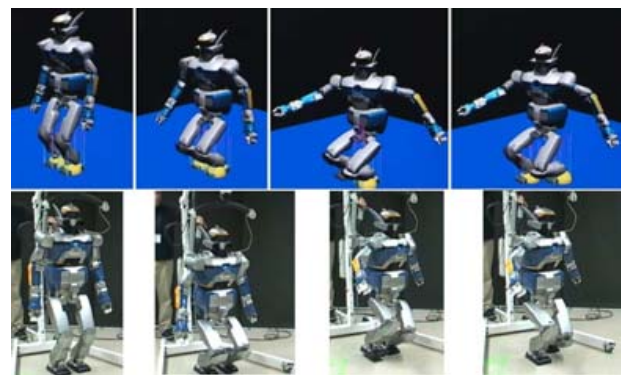

Fig. 5. Simulation and actual one non synchronic step forward.
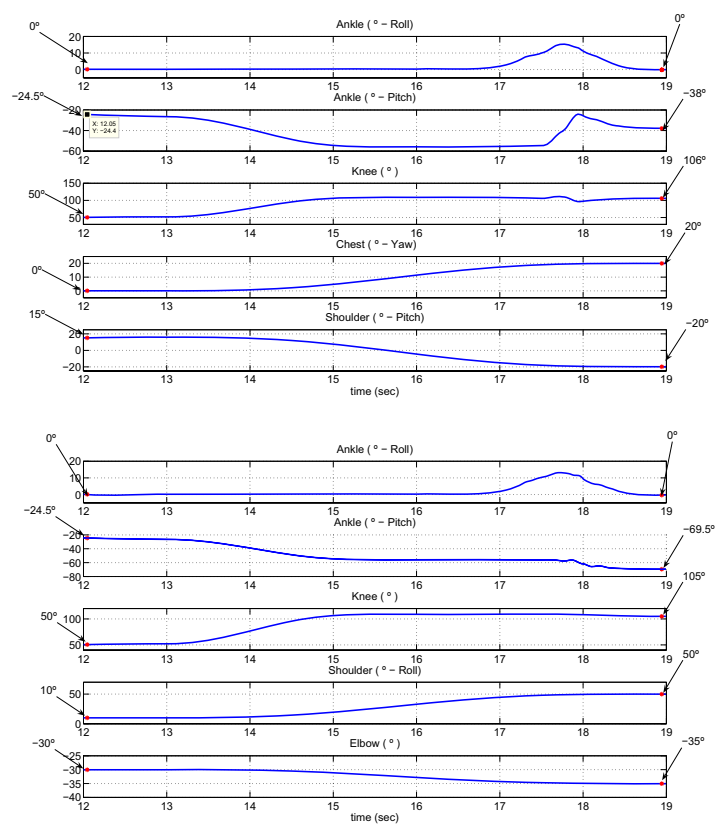

Fig. 6. Actual results of joint angular patterns for doing one acyclic step forward, from the initial to the goal states. In upper curves, the right side of the humanoid is shown; the left side is shown in following curves. In both cases, the goal state is reached by a smooth and natural motion, from the initial one (red dots).

Fig. 5 illustrates, one non sychronic step forward. The following sequences are performed: at first the body gets down from the sitting humanoid robot position and moves its arms, chest and neck; after that, a step is done and it continues moving its arms, chest and neck, to the goal configuration. In Fig. 6, many actual joint evolutions from the initial to the goal states are shown; we can see that the goal state is reached in a smooth and natural way. The 


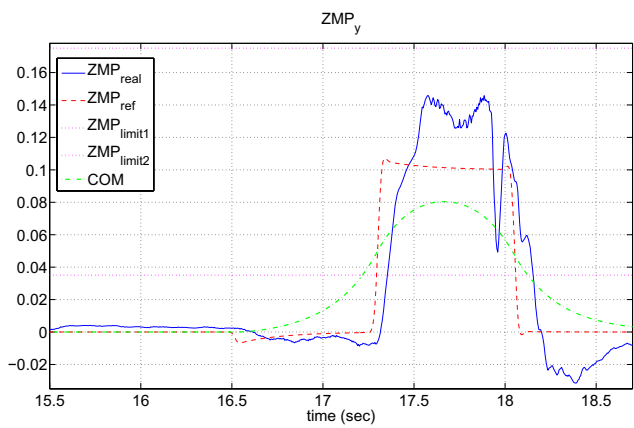

$\mathrm{ZMP}_{x}$
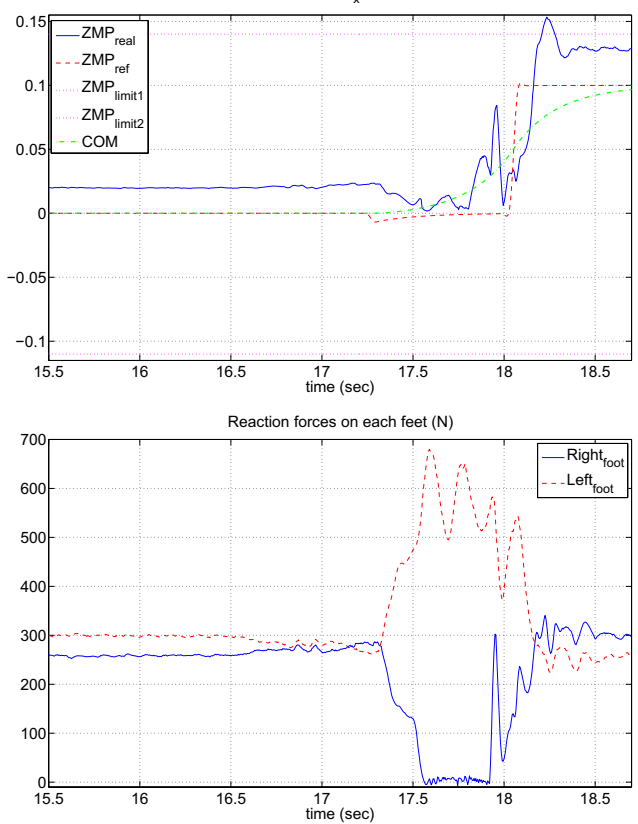

Fig. 7. $Z M P_{y}, Z M P_{x}$ reference and measured. The $Z M P_{\text {limit } 1}$ and $Z M P_{\text {limit } 2}$ are the foot boundaries. $C O M_{y}, C O M_{x}$ trajectory and vertical reaction forces on each foot.

simulation results exhibited a stable dynamic motion. We called it acyclic motion because there is no leg and arm coordination, as compared to normal cyclic walking motion. This simulation is ported and tested successfully on the HRP2 humanoid robot platform (Fig. 5). It is possible to analyze the motion pattern in frontal and sagittal direction (Fig. 7). In the frontal motion direction the actual ZMP (blue line) differs with respect to the reference one (red line), in the single support phase about $0.04 \mathrm{~m}$; but dynamic stability is maintained because it is inside the convex hull. In the sagittal direction for about $0.1 \mathrm{sec}$, the actual ZMP is outside the convex hull, at the start of the single support phase; but the gap is not big enough to lead to unstable motion.

The reaction force on each foot is also analyzed in order to improve the force distribution in the motion pattern. As seen in Fig. 7, during the single support phase, the maximum reaction force on the support foot is about $680 \mathrm{~N}$, nearest

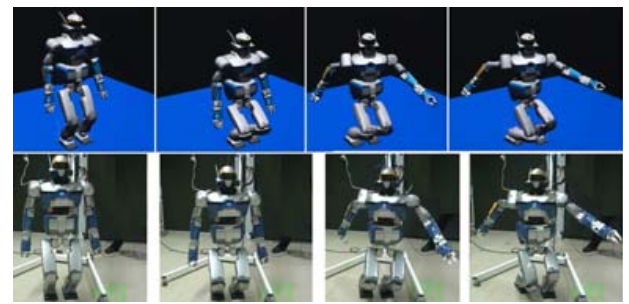

Fig. 8. Simulation and experimental result of one acyclic step backward and rotating.
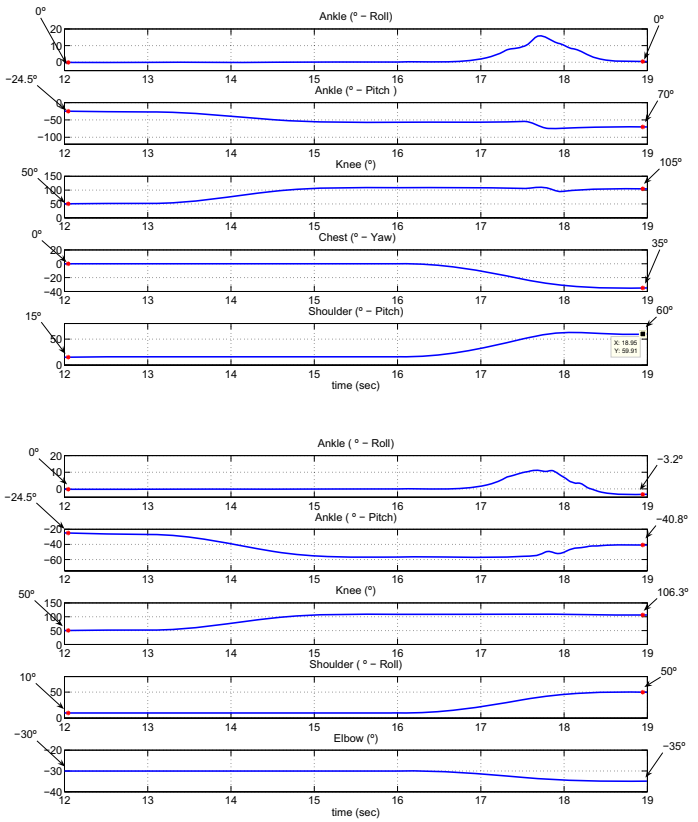

Fig. 9. Actual results of joint angular patterns for doing one acyclic step backward, from the initial to the goal states. In upper curves, the right side of the humanoid is shown; the left side is shown in the following curves. In both cases, the goal state is reached by a smooth and natural motion from the initial one (red dots).

to the humanoid robot weight; and on landing, the reaction force is practically the same on each foot. That is because the motion pattern is designed for assuring equivalent force distribution on each foot.

Let us go to the next case, when the acyclic motion is backward and induces substantial change in the orientation. The simulation is shown in Fig. 8, as the previous experiment, the continuous motion from the sitting position to open arms and down the robot body have been done, when the goal configuration is statically stable too. The joint patterns reach their respective input goal smoothly (Fig. 9).

The proposed motion in the HRP-2 platform (Fig. 8) is successfully validated. The motion patterns and the actual measures can be analyzed, as seen in Fig. 10. In this case, for the single support phase, the maximum ZMP error is about $0.06 \mathrm{~m}$ in frontal motion and $0.025 \mathrm{~m}$ in the sagittal motion. 


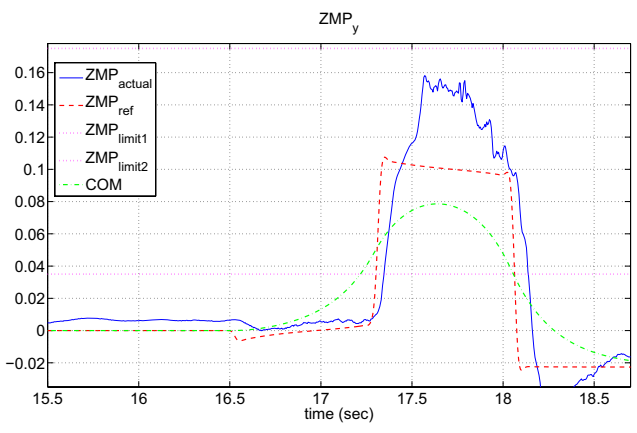

$\mathrm{ZMP}_{\mathrm{x}}$
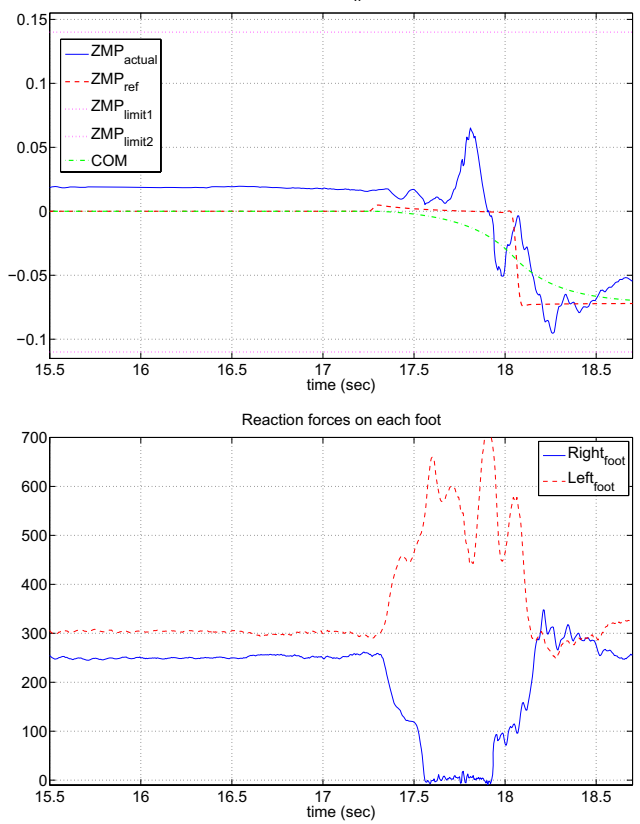

Fig. 10. $Z M P_{y}, Z M P_{x}$ reference and measuring. The $Z M P_{\text {limit } 1}$ and $Z M P_{\text {limit2 }}$ are the foot boundaries. $C O M_{y}, C O M_{x}$ trajectory and vertical reaction forces on each foot.

Hence, the motion is dynamically stable because the actual ZMP is inside the convex hull. Note that the ZMP reference is not strictly tracked by the actual one; this is inherent to our proposed method and is due to the acyclic motion. The reaction forces on each foot are shown in Fig. 10. Their behavior is almost the same as the preview experiment. And the maximum value on the support foot reaches about $700 \mathrm{~N}$, which is approximately the robot weight. Finally, the force distribution on each foot is similar to the starting motion.

The latest case deals with two acyclic steps motion. Simulation results can be seen in Fig. 11. At first, the body is going to the lower its position while the humanoid is taking the first acyclic step. After that, the body goes to the goal configuration while the robot does the second acyclic step. The experiment is validated in the actual humanoid robot (Fig. 12). The actual ZMP is constrained all the time to the support area, in the frontal and sagittal direction, as is shown

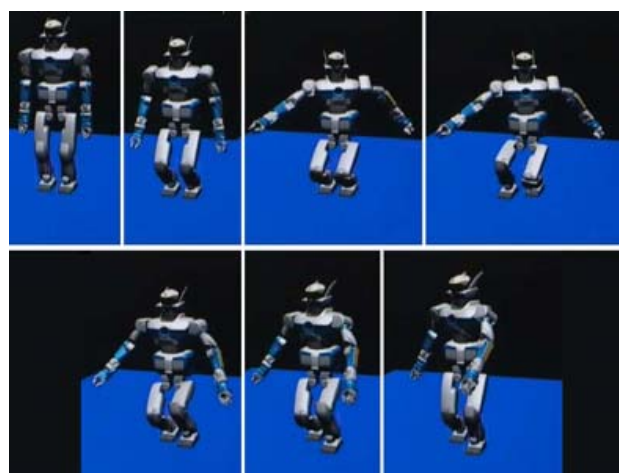

Fig. 11. Simulation of two acyclic step forward

in Fig. 13, when the maximum error is about $0.05 \mathrm{~m}$ in both directions. This way, dynamical stability is obtained. As in the previous cases, the reaction forces on the support feet remains around the weight of the humanoid robot and fair mass distribution is observed on double support. Thus the motion pattern is validated by this fact too.

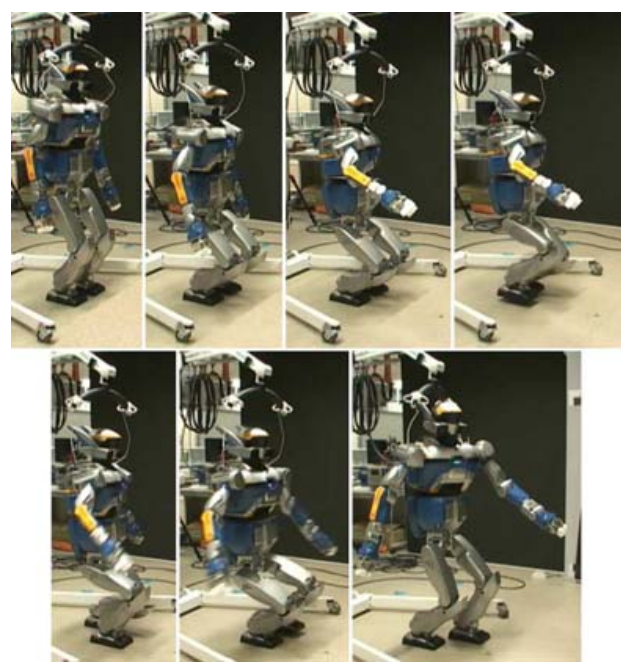

Fig. 12. Experiment of two acyclic step forward

\section{CONCLUSIONS}

A method dealing with non cyclic planar footsteps motion with different body configuration is proposed. It is validated by several simulations and experimental results on the humanoid robot HRP-2. The kinematics proposed is also valid. Hence, the Lie groups, screw theory and Paden-Kahan subproblems prove to be suited for the kinematics and dynamic humanoid robot modeling, which avoids internal singularities and gives us a direct analytical solution. The ZMP Preview Control method appears to be robust enough to be applied even in this case in which height of the COM varies, to generate dynamically stable acyclic motion patterns. Future 


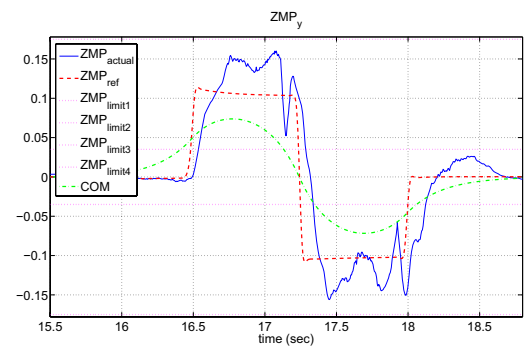

$\mathrm{ZMP}_{\mathrm{x}}$
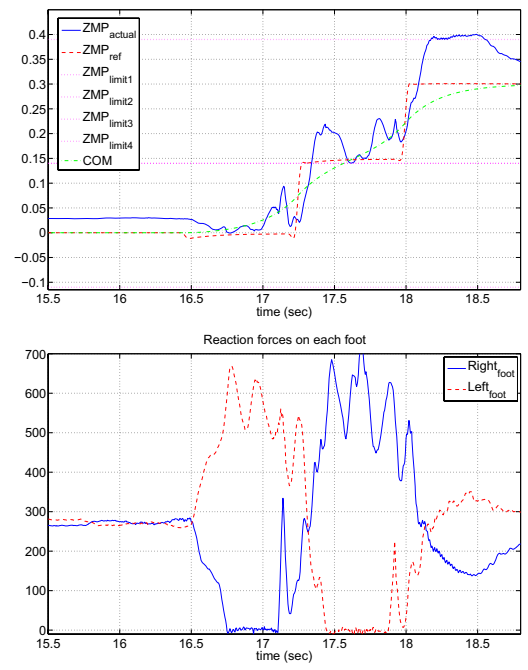

Fig. 13. $Z M P_{y}, Z M P_{x}$ reference and measuring. The $Z M P_{\text {limit } 1}$ to $Z M P_{\text {limit } 4}$ are the feet boundaries. $C O M_{y}, C O M_{x}$ trajectory and vertical reaction forces on each foot.

work is focused in complement the method with autocollision avoidance in order to constraint the motion to physical limits automatically; furthermore to improve the compensation of vetical momentum on rotation motion.

\section{ACKNOWLEDGEMENTS}

First author would like to thank the JRL members for their cooperation and helpful suggestions. This work is partially supported by grants from the ROBOT@CWE EU CEC project, Contract No. 34002 under the 6th Research program www.robot-at-cwe.eu.

\section{REFERENCES}

[1] S. Kajita, F. Kanehiro, K. Kaneko, K. Fujiwara, K. Yokoi, and H. Hirukawa, A realtime pattern generator for biped walking, in IEEE International Conference on Robotics Automation, Washington and DC, May 2002, pp. 3137.

[2] H. Hirukawa, S. Hattori, S. Kajita, K. Harada, K. Kaneko, F. Kanehiro, M. Morisawa, and S. Nakaoka, A pattern generator of humanoid robots walking on a rough terrain, in IEEE International Conference on Robotics and Automation, Roma and Italy, April 10-14 2007, pp. 27812187.

[3] S. Kajita, F. Kanehiro, K. Kaneko, K. Fujiwara, K. Harada, K. Yokoi, and H. Hirukawa, Resolved momentum control: Humanoid motion planning based on the linear and angular momentum, in IEEE/RSJ Intl. Conference on Intelligent Robots and Systems, Las Vegas and Nevada, October 2003, pp. 16441650.
[4] S. Kajita, T. Nagasak, K. Kaneko, K. Yokoi, and K. Tanie, A hop towards running humanoid biped, in IEEE and International Conference on Robotics Automation, New Orleans, LA, April 2004, pp. 629635.

[5] E. S. Neo, K. Yokoi, S. Kajita, and K. Tanie, Whole-body motion generation integrating operators intention and robots autonomy in controlling humanoid robots, IEEE Transactions on robotics, vol. 23, no. 4, pp. 763775, August 2007.

[6] Y. Umetani and K. Yoshida, Resolved motion rate control of space manipulators with generalized jacobian matrix, IEEE Transactions on Robotics, Vol. 5 and No. 3, June 1989

[7] O. Khatib, J. Warren, V. D. Sapio, and L. Sentis, Human-like motion from physiologically-based potential energies, Advances in Robot Kinematics and Kluwer Academic Publishers and first edition and 2004, pp. 149163, 2004.

[8] L. Sentis and O. Khatib, Synthesis of whole-body behaviors through hierarchical control of behavioral primitives, International Journal of Humanoid Robotics, pp. 115, 2005.

[9] N. Mansard, O. Stasse, F. Chaumette, and K. Yokoi, Visually-guided grasping while walking on a humanoid robot, in 2007 IEEE International Conference on Robotics and Automation Roma and Italy and 10-14 April 2007, 2007, pp. 30413047.

[10] E. Yoshida, O. Kanoun, C. Esteves, and J.-P. Laumond, Task-driven support polygon reshaping for humanoids, in Humanoids 2006, 2006.

[11] S. Kajita, F. Kanehiro, K. Kaneko, K. Fujiwara, K. Harada, K. Yokoi, and $\mathrm{H}$. Hirukawa, Biped walking pattern generation by using preview control of zero-moment point, in IEEE International Conference on Robotics Automation, Taipei and Taiwan, September 14-19 2003, pp. 1621626.

[12] B. Verrelst, K. Yokoi, O. Stasse, B. Vanderborght, and H. Arisumi, Mobility of humanoid robots: Stepping over large obstacles dynamically, in IEEE International Conference on Mechatronics and Automation, Luoyang and China, June 25 - 28 2006, pp. 10721079.

[13] P.-B. Wieber, Trajectory free linear model predictive control for stable walking in the presence of strong perturbations, in Humanoids 2006 , 2006, pp. 137142.

[14] T. Katayama, T. Ohkis, T. Tnoues, and T. Kato, Design of an optimal controller for a discrete-time system subject to previewable demand, International Journal of Control, vol. 41, no. 3, pp. 677699, 1985.

[15] R. M. Murray, Z. Li, and S. S. Sastry, Mathematical Introduction to Robotic Manipulation, C. Press, Ed. CRC Press, 1994.

[16] M. Arbulu, J. M. Pardos, L. Cabas, P. Staroverov, D. kaynov, C. Perez, M. Rodriguez, and C. Balaguer, Rh-0 humanoid full size robots control strategy based on the lie logic technique, in 5th IEEE-RAS International Conference

[17] K. Kaneko, F. Kanehiro, S. Kajita, H. Hirukawa, T. Kawasaki, M. Hirata, K. Akachi, and T. Isozumi, Humanoid robot HRP-2, in IEEE International Conference on Robotics Automation, New Orleans and LA, April 2004, pp. 10831090.

[18] M. Vukobratovic and B. Borovac, Zero-moment point - thirty five years of its life, International Journal of Humanoid Robotics, vol. 1, pp. 157 $173,2004$.

[19] M. Arbulu and C. Balaguer, Real-time gait planning for Rh-1 humanoid robot, using local axis gait algorithm, in 7th IEEE-RAS International Conference on Humanoid Robots, Pittsburg, USA, Nov. 29-Dec. 22007.

[20] F. Park, J. Bobrow, , and S. Ploen, A lie group formulation of robot dynamics, Int. J. Robotics Research, vol. 14,No. 6, pp. 609618, 1995.

[21] J. Pardos and C. Balaguer, Rh-0 humanoid robot bipedal locomotion and navigation using lie groups and geometric algorithms, in International Conference on Intelligent Robots and Systems (IROS 2005), Edmonton, Canada, Aug. 2005.

[22] Abraham, Ralph and Marsden, Jerrold E. Foundations of Mechanics, Second Edition. Addison-Wesley Publishing Company, Inc., Redwood City, CA. ISBN 080530102X,(1987)

[23] J. Kuffner, K. Nishiwaki, S. Kagami, M. Inaba, and H. Inoue, Motion Planning for Humanoid Robots, Proc. 11th Intl Symp. of Robotics Research (ISRR 2003), November, 2003. 